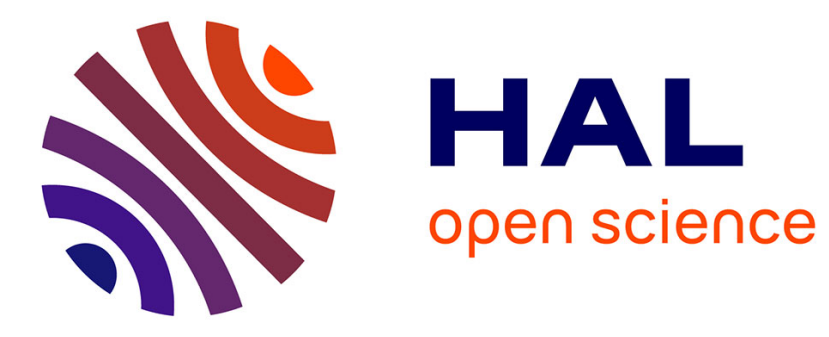

\title{
Le problème de la connaissance dans le "De optima doctrina" de Galien
}

Daniel Béguin

\section{To cite this version:}

Daniel Béguin. Le problème de la connaissance dans le "De optima doctrina" de Galien. Revue des Etudes Grecques, 1995, 108 (Janvier-Juin 1995), pp.107-127. 10.3406/reg · hal-03270187

\section{HAL Id: hal-03270187 \\ https://hal.science/hal-03270187}

Submitted on 24 Jun 2021

HAL is a multi-disciplinary open access archive for the deposit and dissemination of scientific research documents, whether they are published or not. The documents may come from teaching and research institutions in France or abroad, or from public or private research centers.
L'archive ouverte pluridisciplinaire HAL, est destinée au dépôt et à la diffusion de documents scientifiques de niveau recherche, publiés ou non, émanant des établissements d'enseignement et de recherche français ou étrangers, des laboratoires publics ou privés.

$$
\text { Copyright }
$$




\title{
LE PROBLÈME DE LA CONNAISSANCE DANS LE DE OPTIMA DOCTRINA DE GALIEN
}

\author{
DANIEL BÉGUIN
}

\section{BÉGUIN (Daniel), Le problème de la connaissance dans le De optima doctrina de Galien, Revue des Études Grecques, tome 108, Janvier-Juin 1995, p. 107-127.}

\section{RÉSUMÉ}

Dans son opuscule intitulé De optima doctrina, Galien procède à la réfutation du scepticisme tel qu'il est pratiqué chez les Néo-Académiciens. Sa cible est Favorinus d'Arles, un rhéteur qui apprenait à ses étudiants à argumenter successivement le pour et le contre, mais qui les laissait libres d'opter pour la position qu'ils préféraient.

Cela prouve que Favorinus ne fournit à ses étudiants aucun critère certain de jugement, et que lui-même n'a aucun jugement stable. D'ailleurs, dans ses livres, il ne s'est jamais rallié à une position ferme et définitive concernant le statut de la vérité.

Favorinus considère que toutes les perceptions se valent, quel que soit l'état physique ou mental du sujet. Cela est faux, et en même temps contradictoire avec sa propre pratique, puisqu'il estime la position probabiliste des Néo-Académiciens comme étant meilleure que celle des Dogmatiques. En réalité, il faut apprendre à utiliser correctement les perceptions évidentes, comme font ceux qui pratiquent les arts (technai).

Comme l'exercice des perceptions sensibles et intellectuelles n'empêche pas les hommes d'être en désaccord sur de multiples questions, le meilleur maître doit enseigner à ses étudiants une méthode qui soit meilleure que celle des autres. Favorinus ne propose rien de tel.

Donc son enseignement, loin d'être le meilleur, n'est même pas un enseignement du tout. Il n'est pas capable de reconnaître que, parmi les données de la connaissance, certaines sont évidentes et d'autres accessibles par des intermédiaires.

Le fait de mettre en doute les critères du jugement équivaut à en être dépourvu. Galien a écrit un ouvrage intitulé Sur la démonstration, pour apprendre aux étudiants comment parvenir à des connaissances sûres.

\section{ABSTRACT}

In his work entitled De optima doctrina, Galen refutes skepticism as it was practiced among the skeptical period of the Academic school. His target is the sophist Favorinus, a native of Arles, who taught his students to argue for and against successively, but who left them free to choose whatever position they preferred.

This proves that Favorinus does not provide his students with any certain criteria for judgment, and that he himself has no stable judgment. In fact, in his books, he never took a firm and definitive position regarding the status of truth.

Favorinus considers that all perceptions are the same, regardless of the subject's physical or mental state. This is false, and at the same time contradictory with his own practice, since he considers the probabilistic position of the Academic Skeptic school to be better than that of the Dogmatists. In reality, one must learn to use the obvious perceptions correctly, as do those who practice the arts (technai).

Since the exercise of sensitive and intellectual perceptions does not prevent men from disagreeing on many issues, the best teacher should teach his students a method which is better than that of others. Favorinus offers no such thing.

Therefore his teaching, far from being the best, is not even teaching at all. He is not able to recognize that, among the data of knowledge, some are obvious and others accessible through intermediaries.

Doubting the criteria of judgment is the same as lacking those. Galen wrote a book called On Demonstration, to teach students how to achieve reliable knowledge. 
[107] Galien, qui avait bénéficié dans sa jeunesse d'une solide formation philosophique et mathématique ${ }^{1}$, ne s'est pas consacré à la médecine en pur praticien, mais il s'est constamment interrogé sur les méthodes permettant de transformer cet «art»en une connaissance discursive aussi proche que possible d'une science.

Dans l'intense bouillonnement qui caractérisait la vie intellectuelle à Rome au $11^{\mathrm{e}}$ siècle après J.-C., le monde médical était divisé en sectes rivales, très agressives les unes envers les autres, et qui tentaient chacune de justifier le bien-fondé de leur doctrine [108] par des arguments repris aux grands courants philosophiques ${ }^{2}$.

Si les écoles médicales rationalistes ${ }^{3}$ invoquaient l'héritage hippocratique tout en subissant l'influence des Stoïciens (notamment dans le domaine de l'étiologie), on doit cependant constater une montée en puissance du scepticisme chez leurs adversaires. Ainsi, les Empiriques récusaient la raison dans la pratique médicale et rejetaient l'étude des causes, prétendant que le diagnostic se fondait exclusivement sur l'observation de «symptômes » perceptibles par les sens, et que la prescription des remèdes adéquats s'effectuait à partir du souvenir des remèdes appliquées par le médecin ou l'un de ses collègues dans un cas semblable survenu antérieurement ${ }^{4}$. Quant aux Méthodiques, très influents chez les notables romains et très introduits à la cour de l'Empereur, ils n'acceptaient de prendre en compte, pour le traitement des affections, que les causes apparentes aux sens 5 .

[109] Bien qu'il ne fît partie d'aucune école et veillât jalousement à préserver son indépendance intellectuelle ${ }^{6}$, Galien était un rationaliste persuadé qu'on pouvait parvenir, même en médecine, à des connaissances certaines pourvu qu'on les assurât par une

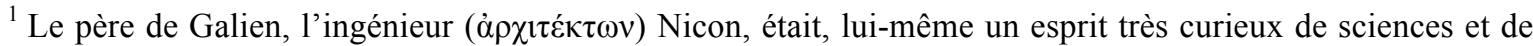
philosophie. Il veilla avec un soin particulier à ce que son fils bénéficiât d'une formation de qualité, pluridisciplinaire, et indépendante de toute école de pensée. Galien parle avec reconnaissance de ce qu'il doit à son père dans le De ordine librorum, 88, 6-22 Müller (= XIX, 59, 1-15 Kühn). On trouvera une traduction de ce passage dans l'ouvrage de Paul Moraux, Galien de Pergame, Belles Lettres, Paris, 1985, p. 41-42. Le détail de cette éducation est exposé dans le De propriorum animi... dignotione et curatione, 28, 9-30, 16 De Boer (= V, 41, 10-45, $1 \mathrm{Kühn).} \mathrm{Le} \mathrm{passage} \mathrm{est} \mathrm{traduit} \mathrm{par} \mathrm{P.} \mathrm{Moraux,} \mathrm{op.} \mathrm{cit.,} \mathrm{p.} \mathrm{42-44.}$

${ }^{2}$ Les doctrines de la secte dogmatique ou logique (c'est-à-dire les écoles médicales rationalistes considérées globalement), de l'empirique et de la méthodique, ainsi que les arguments qu'elles avançaient dans leurs controverses mutuelles, ont été exposés par Galien dans son De sectis ad inlroducendos (éd. G. Helmreich, Claudii Galeni Pergameni scripta minora 3, Teubner, Leipzig, 1893), vraisemblablement rédigé lors de son second séjour à Rome, aux alentours de l'année 175.

${ }^{3}$ Les médecins dogmatiques pensaient qu'il fallait tenir compte de l'état physique du malade, de ses habitudes alimentaires, de son mode de vie, de son environnement climatique et géographique, ainsi que d'une multitude d'autres causes afin d'établir l'indication $\left({ }^{\prime} v \delta \varepsilon 1 \xi 1 \zeta\right)$ du traitement et de le nuancer en fonction de circonstances particulières (âge du patient, saison, etc.). Cf. De sectis ad inlroducendos, chap. Ill, p. 4-6 Helmreich (= I, 69-72 Kühn).

${ }^{4}$ L'école médicale empirique était l'héritière du nihilisme étiologique professé par Philinos de Cos, disciple d'Hérophile, qui la fonda vers 250 av. J.-C. Elle réduisait la connaissance médicale à l'accumulation d'observations et à l'expérience vécue par le praticien. Cf. De sectis ad inlroducendos, chap. Il, p. 2-4 Helmreich (= I, 66-69 Kühn).

${ }^{5}$ Fondée (ou plutôt réorganisée) par Thessalos de Tralles du temps de Néron, la secte méthodique s'appuyait sur une synthèse curieuse de scepticisme et de matérialisme atomistique. Elle enseignait que les affections étaint dues à des déséquilibres dans l'état du tissu corporel (ou diathèse). Il y avait trois états, le relâchement, le resserrement et le mixte, un composé des deux premiers. Quand le médecin déterminait que la cause de l'affection était un relâchement (généralement identifié avec un excès de sécrétions), il prescrivait un traitement produisant un resserrement. S'il y avait resserrement (souvent confondu avec la rétention des sécrétions normales), il tâchait de provoquer un relâchement. Dans le cas d'un état mixte, il combattait celle des deux composantes qui lui paraissait la plus dangereuse.

${ }^{6}$ Cf. De locis affectis, VIII, 142, 17-144, 15 Kühn (traduit par P. Moraux, op. cit., p. 48-49).
} 
méthode démonstrative inspirée de la démarche géométrique ${ }^{7}$. Cette conception est exposée à l'occasion de rappels et de brèves remarques dispersés dans l'ensemble de son œuvre. Elle apparaît également sous forme d'une argumentation suivie dans un bref opuscule intitulé De optima docirina (parfois appelé De optimo docendi genere) ${ }^{8}$.

Pour réfuter un éminent représentant des thèses probabilistes de l'école néoacadémique, Favorinus d'Arles, Galien est amené à développer ses idées concernanl la possibilité de la connaissance, la nature innée ou acquise de celle-ci, les différentes sortes de critères qui mènent à la vérité et à s'interroger sur le rôle de l'enseignant par rapport à l'étudiant.

L'Histoire n'a guère conservé le souvenir de Favorinus d'Arles, au point que nous ignorons la date de sa naissance et celle de sa mort : A. Barigazzi, l'éditeur de ses œuvres, estime qu'il a vécu entre les années 80 et 160. Mais il était considéré par ses contemporains comme un homme remarquable. Élève du rhéteur Dion de Pruse, ami d'Hérode Atticus et de Plutarque, il fut lui-même un rhéteur et un érudit, une des personnalités les plus représentatives de la culture à Rome au milieu du $\mathrm{n}^{\mathrm{e}}$ siècle après J.-C. Nous n'avons conservé que des fragments et deux discours entiers ${ }^{9}$, soit en tout vingt-cinq titres, d'une œuvre qui aurait voulu, dit-on, rivaliser avec la production littéraire de Plutarque (plus de 125 titres).

[110] Très attiré par la spéculation philosophique, Favorinus défendit les positions de l'école néo-académique ${ }^{10}$. Aulu-Gelle, son disciple, le tenait pour un philosophe d'envergure au point d'en faire un des protagonistes de ses Nuits attiques. D'après Diogène Laërce, il aurait écrit un ouvrage, Sur les tropes pyrrhoniens, dans lequel il modifiait la classification des dix tropes de la suspension du jugement établie par Ænésidème. Il a servi de source pour les notices doxographiques de Diogène Laërce ${ }^{11}$ grâce à son Histoire variée

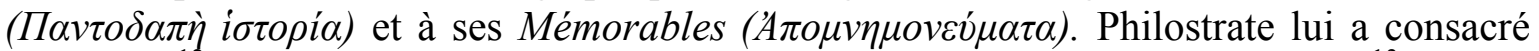
une notice $^{12}$ dans sa Vie des sophistes (I, 8) ainsi que Suidas dans son dictionnaire ${ }^{13}$.

Favorinus fut-il davantage qu'un simple compilateur? Lucien se moque de ses prétentions philosophiques dans le Demonax et dans L'Eunuque, mais il l'attaque davantage sur son physique que sur ses idées ${ }^{14}$. Galien, quant à lui, nous apprend qu'il a rédigé, dans le but de combattre les positions de Favorinus, outre le De optima doclrina,

\footnotetext{
${ }^{7}$ Cf. De libris propriis, 115, 21 - 117, 16 Müller (=XIX, 39, 1 -41, 6 Kühn). Le passage est traduit par P. Moraux, op. cit., p. 44-46.

${ }^{8}$ Cet opuscule a fait l'objet d'une édition scientifique : Adelmo Barigazzi, Galeni De optimo docendi genere Exhortatio ad medicinam (Protrepticus), CMG V, 1,1, Berlin, 1991. Le texte grec est accompagné d'un apparat critique, d'une introduction en latin et d'une traduction en italien.

${ }^{9}$ Ce qui subsiste de Favorinus a été rassemblé dans A. Barigazzi, Favorino di Arelate, opéré, Felice Le Monnier, Firenze, 1966.

${ }^{10}$ Cf. les témoignages d'Aulu-Gelle (N.A. XX, 1, 9.21) et de Galien (De optima doclrina, I, 1-2). Lucien, quant à lui, met en scène Favorinus d'une façon bouffonne dans son Eunuque et parle au chapitre 7 d'un

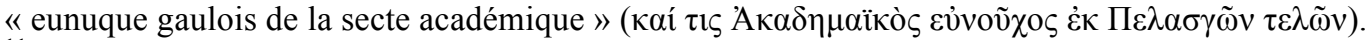

${ }^{11}$ On trouvera dans l'ouvrage d'A. Delattre, La vie de Pythagore de Diogène Laërce, Lemertin, Bruxelles, 1922, p. 46-47, la démonstration que Favorinus était une des sources utilisées par Diogène Laërce.

12 Philostrate, Vita sophistarum, éd. Wilmer Gave Wright, Loeb, London, 1922, p. 22-29.

${ }^{13}$ Suidae lexicon, Pars IV, éd. Ada Adler, Teubner, Leipzig, 1935, p. 690, 1. 16-26.

${ }^{14}$ Favorinus était né hermaphrodite. Ce détail a inspiré quelques railleries au Cynique Demonax (Demonax 12 et 13). Dans l'Eunuque, Lucien représente Favorinus sous les traits, à peine déguisés, de l'eunuque Bagoas se disputant avec un autre philosophe dans un procès pour l'obtention d'un poste de professeur de philosophie rétribué par l'Empereur.
} 
deux ouvrages aujourd'hui perdus. L'un ${ }^{15}$, qui s'intitulait Pour Epiclète, contre Favorinus

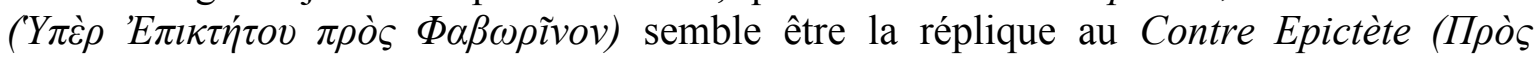

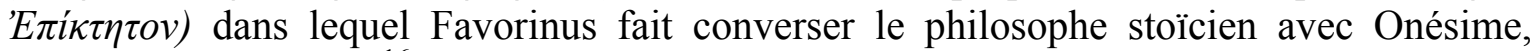

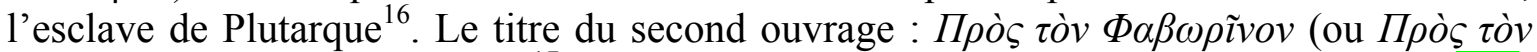
$\Phi \alpha \beta \omega \rho$ ivov d'après Brinkmann ${ }^{17}$ et Barigazzi) $\kappa \alpha \tau \grave{\alpha} \Sigma \omega \kappa \rho \alpha ́ \tau o v \varsigma$ fait difficulté, et cela [111] d'autant plus que nous ne pouvons en reconstituer le contenu ${ }^{18}$. Dans la mesure où ce dernier pourrait être la réponse à un livre de Favorinus intitulé Sur Socrate et son opinion

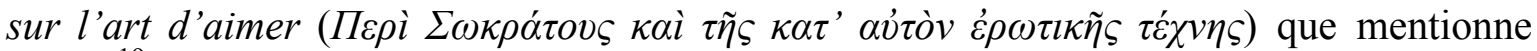
Suidas ${ }^{19}$, et sachant par ailleurs que Favorinus considérait Socrate comme un modèle à imiter $^{20}$, il conviendrait de le traduire par Contre l'ouvrage de Favorinus concernant Socrate $^{21}$.

Le texte du De optima doclrina comporte quelques passages difficiles qui dissimulent peut-être des lacunes, et que l'éditeur allemand J. Marquardt a systématiquement considérés comme des interpolations ${ }^{22}$. L'opuscule, dont la date de rédaction est inconnue, semble avoir été composé entre 162, date à laquelle Galien revint pour la seconde fois à Home, et 166, date de son retour à Pergame. Favorinus était-il encore vivant lorsque l'opuscule a été rédigé ? Au cours de la discussion ${ }^{23}$, Galien emploie

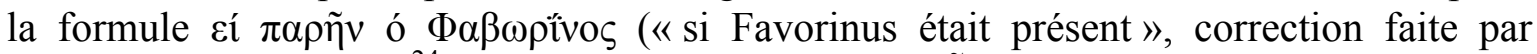

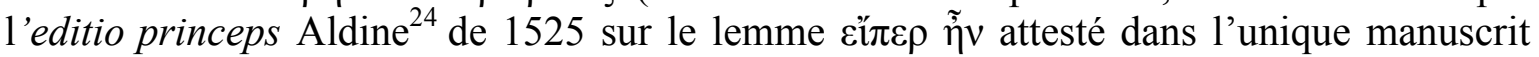

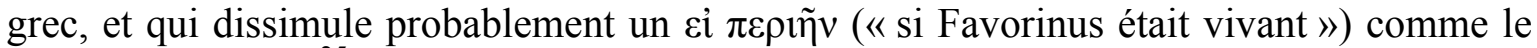
suggère Goulston ${ }^{25}$ dans son Opuscula varia (1640). Mais indépendamment de ces considérations, on estime que Favorinus est mort aux alentours de l'an 160.

L'unique témoin grec actuellement disponible est le Laurentianus plut. 74, 3 qui date de la fin du XII ${ }^{\mathrm{e}}$ siècle. Nicolas de Rhegium fit de l'opuscule une traduction latine, plusieurs fois réimprimée ${ }^{26}$, qui semblerait reposer sur un modèle différent, à moins que Nicolas ait pris quelques libertés dans sa traduction.

[112] Examinons maintenant le contenu du De optima doctrina. Il est traditionnellement divisé en cinq parties depuis l'édition Chartier ${ }^{27}$ de 1639 . Le fil du discours galénique est le suivant.

(a) Favorinus affirme que le meilleur enseignement consiste à apprendre aux étudiants à argumenter le pour et le contre, avant de les laisser libres de se rallier par euxmêmes à la position qu'ils estiment vraie. Mais à quoi sert cet apprentissage puisque

${ }^{15}$ CF. De libris propriis, 120, 6 Müller (= XIX, 44 Kühn).

${ }^{16} \mathrm{Cf}$. De optima doctrina, 92, 13-14 Barigazzi (= I, 41, 5-6 Kühn).

${ }^{17}$ August Brinkmann, Galeni De optimo docendi genere libellus, Univ. Progr. Bonn, 1914.

${ }^{18}$ Cf. De libris propriis, 121, 10-11 Müller (= XIX, 45, 12 Kühn).

${ }^{19}$ Suidae Lexicon, 690, 23-25.

${ }^{20}$ Cf. la discussion de Barigazzi dans Favorino di Arelate, p. 76.

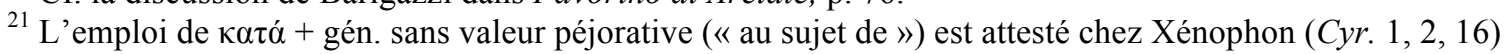

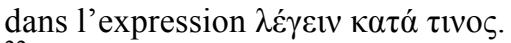

22 Johann Marquardt, Claudii Galeni Pergameni scripta minora 1, Teubner, Leipzig, 1891.

${ }^{23}$ Cf. 98,10 Barigazzi (= I, 45, 15 Kühn).

${ }^{24}$ Galeni librorum pars prima [quinla], Venetiis, Aldus et A. Asulanus, 1525 (5 vol.).

${ }^{25}$ Theodore Goulston, Claudii Galeni pergameni opuscula varia..., Londini, R. Balger-Ph. Stephanus-Ch. Meredith, 1640.

${ }^{26}$ La traduction latine de Nicolas parut d'abord en 1490 dans l'édition latine que Diomedes Bonardus donna des oeuvres de Galien à partir de sources médiévales. Elle fut réimprimée en 1515 dans l'édition latine des œuvres complètes de Galien publiée à Pavie par Rusticus Placentinus, puis encore en 1522 et en 1528 chez les Juntes.

${ }^{27}$ René Chartier, Hippocratis Coi el Claudii Galeni Pergameni... opera, vol. 1, t. 2, Lutetiae Parisiorum, 1639. La date de 1679 correspond à la parution des derniers volumes de l'ensemble des œuvres complètes. 
Favorinus ne fournit à ses étudiants aucun critère de jugement et que lui-même n'a pas de jugement stable ? Car si, dans certains ouvrages, il semble admettre la possibilité d'une connaissance sûre, dans d'autres, au contraire, il soutient que cette dernière n'existe pas.

(b) Nous considérons généralement que nos perceptions ne sont pas toutes vraies : celles des hommes en bonne santé physique et mentale, ou en état de veille, sont plus crédibles que celles des dormeurs ou des personnes malades physiquement ou mentalement. Si toutes les perceptions se valaient, personne ne pourrait juger le vrai. De plus, Favorinus n'aurait aucune raison de privilégier l'enseignement des Académiciens par rapport à celui des Dogmatiques. Mais si l'on admet que la connaissance repose sur les perceptions effectuées à l'aide des critères naturels, le véritable enseignement dont on a besoin est celui qu'on utilise dans les «arts »: en s'appuyant sur les perceptions évidentes, le maître exerce l'apprenti à réussir ses exercices et à corriger ses erreurs. Ceux qui, au contraire, habituent leurs étudiants à se méfier des évidences et qui dégagent des problèmes sans leur donner de solution ne sont que des sophistes.

(c) Lorsqu'il faut examiner la justesse des raisonnements, les dispositions naturelles ne suffisent pas, car tous les hommes ne sont pas d'accord sur les mêmes questions. Il est donc nécessaire d'apprendre une méthode et de s'y exercer, comme on fait dans les « arts », avant de procéder à cet examen. Or, les Académiciens n'ont jamais proposé une telle méthode : ils demandent à leurs étudiants de juger sans leur fournir les instruments du jugement. Prétendre que la situation est différente dans les [113] «arts» et dans les matières abstraites est une ineptie. Refuser de reconnaître l'existence d'un critère du vrai et du faux, et d'apprendre à s'en servir, c'est se refuser l'accès à la connaissance et se condamner au silence.

(d) Ainsi donc, le genre d'enseignement que propose Favorinus, loin d'être le meilleur, n'est même pas un enseignement du tout. Il faut d'abord apprendre à juger en utilisant les critères naturels que sont les sens, car seuls les sens permettent de saisir les réalités sensibles. De même, seul l'esprit permet de saisir les réalités intelligibles. Ce qui est évident aux sens ou à l'esprit ne nécessite pas d'examen complémentaire ; en revanche, ce qui n'est pas évident se perçoit par un intermédiaire. Il existe des méthodes, que Galien se fait fort d'enseigner, suivant lesquelles on appréhende ce qui n'est pas évident ou grâce auxquelles on juge les connaissances acquises.

(e) L'attitude de Favorinus revient à vouloir juger sans en avoir le moyen. Pire, c'est la même chose de se défier des critères du jugement que d'en être dépourvu comme les ânes. Le véritable enseignant, a dit Platon, est celui qui guide dans la contemplation des choses ceux dont la vue est moins perçante que la sienne. Un livre Sur la démonstration a été écrit pour enseigner aux étudiants comment acquérir des connaissances sûres en suivant une méthode démonstrative.

Il est clair, d'après le résumé précédent, que Galien s'attaque à l'approche sceptique de la connaissance, mais on aurait tort de croire qu'il confond indistinctement tous les partisans du scepticisme. Le De optima doctrina discerne nettement, dans le camp des adversaires de la connaissance certaine, les Anciens (ot $\pi \alpha \lambda \alpha$ ió $\tau \varepsilon \rho o \imath$ ) et les Modernes (oí

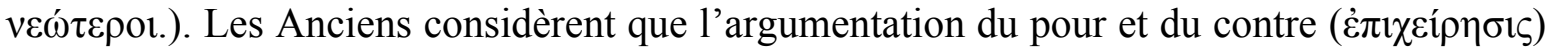

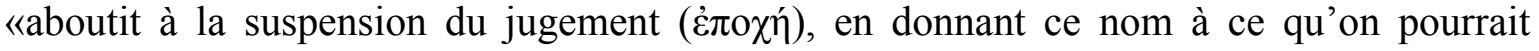
appeler l'indétermination, c'est-à-dire l'incapacité de rien définir ou d'émettre un avis certain $^{28}$. Il s'agit donc des premiers représentants de la nouvelle Académie, disciples

\footnotetext{
${ }^{28}$ Cf. 92, 5-7 Barigazzi (= I, 40, 3-7 Kühn).
} 
d'Arcésilas (315-240 av. J.-C.) dont les conceptions ont subi l'influence du scepticisme de Pyrrhon. Le nom de Pyrrhoniens est d'ailleurs employé un peu plus loin dans l'opuscule, en parallèle [114] avec celui des Académiciens ${ }^{29}$, ce qui montre que Galien établit une séparation entre les deux courants philosophiques. Néanmoins, l'argumentation du De optima doctrina, en tant qu'elle réduit la doctrine des anciens néo-Académiciens à la seule suspension du jugement, tend à les confondre avec les Pyrrhoniens, partisans du scepticisme intégral.

Les modernes néo-Académiciens, quant à eux, sont définis de la manière suivante : «Mais les Modernes, car Favorinus n'est pas le seul à se comporter ainsi, poussent certaines fois la suspension du jugement si loin qu'ils n'admettent, même pas que le soleil est compréhensible ( $\kappa \alpha \tau \alpha \lambda \eta \pi \tau o ́ \varsigma)$, et d'autres fois donnent une telle extension à la faculté de juger qu'ils la laissent librement s'exercer chez leurs étudiants sans leur avoir, au

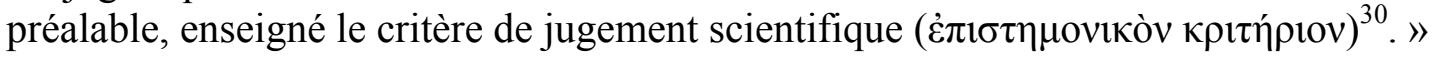

Suivant cette présentation, les Modernes oscillent entre le scepticisme intégral, niant l'évidence sensible, et l'attitude qui consiste à donner au jugement une totale liberté d'action en dehors de tout encadrement doctrinal ou magistral. Galien vise la seconde génération de néo-Académiens, dont Favorinus défend les positions, et dont la pratique

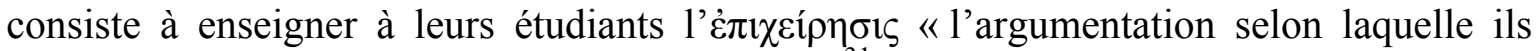
soutiennent alternativement les thèses opposées ${ }^{31}$ » " tout en "laissant leurs étudiants libres de se rallier par eux-mêmes à celles qui son vraies ${ }^{32} \gg$. La doctrine visée est donc le probabilisme qui renvoie dos à dos la thèse et l'antithèse au nom de l'égale vraisemblance des deux positions. Voilà pourquoi, dans une de ses attaques contre les Académiciens, Galien s'en prend nommément à Carnéade (219-129 av. J.-C.) ${ }^{33}$.

Le fait que Galien concentre ses coups sur les modernes néo-Académiciens, en prenant Favorinus pour cible principale, ne signifie nullement qu'il affectionne, $a$ contrario, les anciens néo-Académiciens ou les Pyrrhoniens. Même si nous ne disposons pas d'un ouvrage analogue au De optima doctrina et qui serait spécifiquement dirigé contre un représentant de l'école d'Arcésilas [115] ou appartenant à la mouvance de Pyrrhon, en revanche, nous trouvons dans l'ensemble du corpus galénique un nombre suffisant de remarques montrant à quel point le médecin de Pergame éprouvait à l'égard des Pyrrhoniens, et de ceux qui en reprenaient les idées, un mélange de haine et de mépris.

Évoquant dans le De libris propriis la manière dont il s'était formé à la philosophie dans sa jeunesse, Galien parle des maîtres péripatéticiens et stoïciens dont il avait suivi les leçons. Comme ceux-ci enseignaient des "théorèmes logiques » que leur jeune auditeur trouvait peu utiles, voire contradictoires d'une école à l'autre ou même avec les données élémentaires de la physique, peu s'en fallut qu'il ne sombrât dans un «scepticisme pyrrhonien », s’il n'avait découvert à temps les mathématiques pour le sauver du naufrage intellectuel ${ }^{34}$.

Symptôme de la défaite ou de la démission de la raison, le pyrrhonisme guette ceux dont la doctrine laisse place à des contradictions non résolues ou se montre incapable

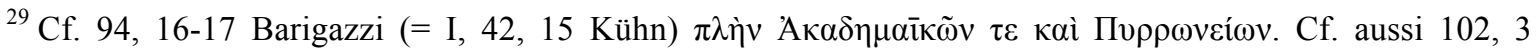
Barigazzi (= I, 48,5 Kühn).

${ }^{30}$ Cf. 92, 7-11 Barigazzi (= I, 40, 7-41, 2 Kühn).

${ }^{31}$ Cf. 92, 2-3 Barigazzi (= I, 40, 3 Kühn).

${ }^{32}$ Cf. 92, 16-17 Barigazzi (= I, 41, 9-10 Kühn).

${ }^{33}$ Cf. 96, 21 Barigazzi (= I, 45, 1-2 Kühn).

${ }^{34}$ Cf. De libris propriis, 116, 12-33 Millier (= XIX, 39, 1-41,7 Kühn). La passage est traduit dans P. Moraux, op. cil., p. 45.
} 
d'expliquer des faits concrets. Lorsque les Érasistratéens renoncent à répondre aux arguments de leurs adversaires concernant le mécanisme de la circulation du sang, ils trouvent refuge «dans la cambrousse pyrrhonienne comme auprès d'un autel des suppliants $»^{35}$. De même, en refusant toute étiologie qui leur permettrait d'expliquer pourquoi l'estomac se contracte au contact des aliments et pourquoi les veines produisent du sang, sous prétexte que ce genre de recherche relève de la philosophie de la nature et non pas de la médecine, les Érasistratéens se comportent comme des « douteurs et des Pyrrhoniens ${ }^{36}$. Galien considère donc que la possession d'un corps de doctrine, permettant d'expliquer le comment et le pourquoi des choses en posant des affirmations et en réfutant victorieusement l'argumentation adverse, est un constituant nécessaire de la connaissance médicale.

Pour qualifier l'attitude de ces Sceptiques qui n'acceptent que le doute, qui se défient des témoignages de la sensation et qui prétendent ne pas même percevoir leurs propres

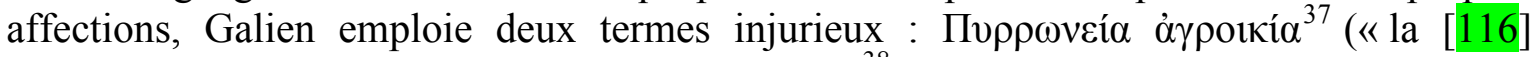

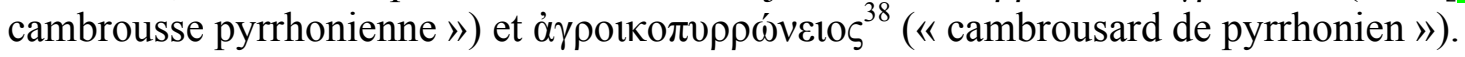

Si Galien s'en prend à Favorinus, et à travers lui aux modernes néo-Académiciens, non pas avec de simples invectives, mais en développant une argumentation, c'est que le doute subtil des probabilistes, qui tend à faire accroire que tout est acceptable, lui parait plus insidieux que l'attitude trop grossièrement monolithique des Pyrrhoniens et de leurs émules, qui suggère que rien n'est acceptable.

Avant d'attaquer Favorinus sur des points précis de doctrine, Galien se fait un malin plaisir de montrer que son adversaire n'a pas de doctrine stable. Au début du De optima doctrina se trouve un bref rappe ${ }^{39}$ du titre de six œuvres écrites par Favorinus et du sens général de cinq d'entre elles. Ce dernier expose partout la même position philosophique : il n'existe aucune connaissance certaine, on peut soutenir le pour et le contre sur n'importe quelle question, et c'est à chacun (notamment aux étudiants) de se faire librement sa propre opinion. Mais la conclusion à laquelle parvient Favorinus varie fortement d'un ouvrage à l'autre.

Voici l'ordre de présentation adopté par Galien : dans son Plutarque, Favorinus penche vers la probabilité d'une connaissance certaine; dans son Alcibiade, il considère que rien n'est connaissable avec certitude; dans trois ouvrages qu'il a intitulés Sur la représentation compréhensive et qu'il a écrits l'un contre Adrien, le second contre Dryson et le troisième contre Aristarque, il soutient avec vigueur que la connaissance certaine n'existe pas. Galien mentionne aussi le Contre Épictète qui prend place entre le Plutarque et l'Alcibiade. Mais on ne peut rien déduire sur son contenu car il est impossible de déterminer si la formule «il réitère son affirmation dans son Contre Épictète » s'applique à la totalité de l'argumentation développée dans le Plutarque ou seulement à la nécessité de laisser les étudiants juger par eux-mêmes. [117]

Il semble exister une logique interne dans l'oscillation de la pensée de Favorinus. En supposant que Galien ait présenté les ouvrages du rhéteur d'Arles en respectant l'ordre

\footnotetext{
${ }^{35} \mathrm{Cf}$. An in arleriis natura sanguis continealur, IV, 727, 9-12 Kühn.

${ }^{36} \mathrm{Cf}$. De naluralibus facullatibus, II, 126, 7-127, 5 Kühn.

${ }^{37} \mathrm{Cf}$. An in arleriis nalura sanguis conlinealur, IV, 727, 10-11 Kühn.

${ }^{38} \mathrm{Cf}$. De differentiis pulsuum, VII, 711,3 Kühn et De praenolione ad Epigenem, XIV, 628, 18 Kühn. Ce terme et le précédent, qui sont attestés seulement chez Galien, semblent être des néologismes forgés en vue de la polémique, comme cela se faisait en ce temps-là lors des disputes philosophiques et scientifiques.

${ }^{39}$ Cf. 92, 1 -94, 9 Barigazzi (= I, 41, 2-42, 6 Kühn).
} 
chronologique, on peut émettre l'hypothèse suivante : celui-ci aurait d'abord incliné vers un probabilisme «positif», admettant la possibilité d'une connaissance certaine (Plutarque); puis il aurait évolué jusqu'à un point où ses tendances positives et négatives se seraient équilibrées, lui faisant estimer qu'on ne peut trancher ni dans un sens ni dans l'autre (Alcibiade) ; finalement, cet équilibre étant rompu, il aurait basculé vers un scepticisme "négatif », le poussant à affirmer l'impossibilité de la connaissance certaine (ses trois ouvrages Sur la représentation compréhensive).

Mais puisque les ouvrages susmentionnés sont perdus, nous en sommes réduits aux hypothèses. Ce qui est sûr en revanche, c'est que Galien présente son adversaire comme une girouette qui oscille, au gré des circonstances, entre des positions différentes, voire opposées. C'est le symptôme qu'un probabiliste ne possède aucune doctrine solide, qu'il n'hésite pas à se contredire au mépris des règles fondamentales de la logique discursive, bref, qu'il dit n'importe quoi.

Non seulement Favorinus se montre inconséquent sur le plan des idées, mais il n'est pas non plus très rigoureux dans le choix de sa terminologie. Le rhéteur d'Arles était renommé pour s'exprimer dans un style aussi proche que possible de la pureté attique. Mais alors, s'étonne Galien ${ }^{40}$, pourquoi employer les termes de "compréhensible »

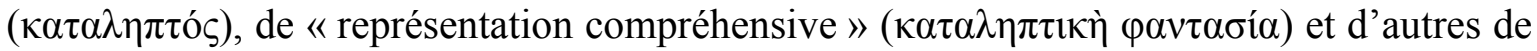
la même famille ? En effet, le langage commun préfère au terme stoïcien utilisé pour

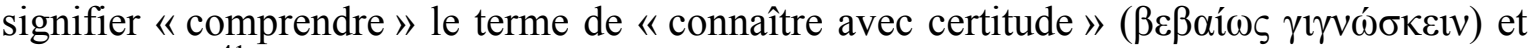
ainsi de suite ${ }^{41}$.

Certes, il s'agit chez Galien d'un lieu commun que de se moquer des partisans des sectes qui changent les mots sans se rendre compte qu'ils sont d'accord sur les réalités ${ }^{42}$, ou qui forgent des mots nouveaux parce qu'ils ne savent pas le grec. Le médecin de Pergame affirme qu'il utilise les mots courants, plutôt que les termes techniques, sauf s'il y a risque de confusion. . [118] Certains commentateurs ont vu dans cette attitude un principe conservateur, mais l'enjeu est plus important

Adopter une terminologie, surtout si elle fait un usage fréquent des néologismes et des termes techniques, c'est adhérer implicitement à la doctrine de l'école qui l'a forgée.

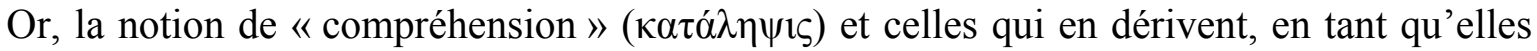
reprennent des mots anciens en leur affectant un sens nouveau, sont une création des Stoïciens ${ }^{43}$. Les vrais Sceptiques, au témoignage de Sextus Empiricus ${ }^{44}$, nient la théorie de la connaissance stoïcienne et donc toute forme de compréhension.

Même si Favorinus emploie ces termes pour démontrer que la notion qu'ils sont censés décrire est inexistante ( $\alpha v v ́ \pi \alpha \rho \kappa \tau o \varsigma)$, Galien considère qu'il y a un sérieux paradoxe, de la part d'un probabiliste, à utiliser quasi naturellement un vocabulaire appartenant à ces dogmatiques que sont les Stoïciens.

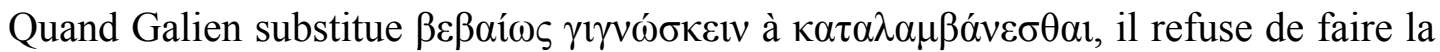
moindre concession, serait-elle de vocabulaire, à une école philosophique dont il n'admet

\footnotetext{
${ }^{40}$ Cf. 94, 1-6 Barigazzi (= I, 41, 14-42, 1 Kühn).

${ }^{41}$ Cf. 94, 11 Barigazzi (= I, 42, 9 Kühn).

${ }^{42}$ Cf. Quod optimus medicus sit quoque philosophus, 28, 8-29, 3 Müller (= I, 61. 16-62, 7 Kühn).

${ }^{43}$ Pour faire mieux saisir par ses auditeurs les différentes étapes menant de la perception simple à la compréhension, Zénon de Citium illustrait son propos à l'aide de mouvements de la main : cf. Cicéron, Académiques, II, 47, 145 (= Arnim, SVF, I, 19, 66).

${ }^{44}$ Selon les Hypotyposes pyrrhoniennes (I, 201), la formule ov̉ $\kappa \alpha \tau \alpha \lambda \alpha \mu \beta \alpha ́ v \omega$ (« je ne comprends pas ») est l'une de celles qui servent à caractériser l'attitude sceptique. La notion de représentation compréhensive est attaquée en détail dans Hypotyposes pyrrhoniennes, II, 70-78.
} 
ni la doctrine ni les «théorèmes logiques ». Chez Favorinus au contraire, le flottement de la doctrine va de pair avec le laxisme de la terminologie. C'est un sceptique infidèle qui trahit plus d'une fois les règles de conduite du vrai sceptique.

Telles sont les remarques préliminaires que nous livre Galien avant d'attaquer plus précisément la doctrine de Favorinus.

La connaissance est-elle possible? Pour répondre à cette question d'une façon simple et non dogmatique, Galien tire ses exemples de la vie courante. Il remarque que nous faisons spontanément la distinction entre les perceptions fausses et les vraies. Les premières concernent « les choses que nous croyons voir, entendre ou plus généralement percevoir par nos sens $»^{45}$, tandis que les secondes s'appliquent aux choses que «nous [119] voyons ou plus généralement percevons véritablement ». Les critères permettant de distinguer les perceptions réelles des semblants de perception sont d'ordre physiologique : l'état du corps (santé ou maladie), l'état de l'esprit (normalité ou folie) et l'activité de la personne (éveil ou sommeil).

On reconnaît ici les exemples utilisés par les Sceptiques pour justifier la suspension du jugement. Les Hypotyposes pyrrhoniennes se fondent précisément sur la différence de perception des sons, des odeurs, du toucher, des couleurs, du goût entre les malades mentaux et les gens normaux pour montrer que nos perceptions, et par conséquent les jugements qui en découlent, dépendent de "circonstances extérieures» (I, 101). L'argument est repris en I, 103 avec l'exemple des malades et des bien portants, et en I, 104 avec celui des dormeurs et des personnes éveillées.

Le Sceptique constate l'opposition irréductible des perceptions et ne cherche pas à la dépasser car, remarque-t-il, chaque individu est intimement persuadé de la validité de ses perceptions : le fou croit autant à ce qu'il voit que l'homme sensé, et ainsi de suite car chaque individu est soumis à des circonstances extérieures dont l'effet varie d'une personne à l'autre. "Chacun faisant partie du procès», personne ne peut être juge impartial des objets extérieurs, car chacun est "troublé » par les circonstances dans lesquelles il est plongé ${ }^{46}$. Sextus Empiricus construit son aporie sur une base solipsiste : chacun est enfermé dans son individualité et il n'existe aucune instance supérieure pour trancher entre les différences de jugement.

L'argument galénique brise la difficulté en réinsérant l'individu dans une pluralité humaine. Il existe un ensemble de personnes : $\pi \alpha ́ v \tau \varepsilon \varsigma$ óv $\theta \rho \omega \pi \mathrm{ol}^{47}$ qui sont d'accord sur certaines choses, à l'exception d'un groupe minoritaire d' "Académiciens » et de «Pyrrhoniens ». La notion de fou et d'homme sensé, de malade et de bien portant, de dormeur et d'homme éveillé repose sur un consensus, à partir duquel on peut désormais distinguer entre les perceptions vraies et les fausses. C'est également cette communauté

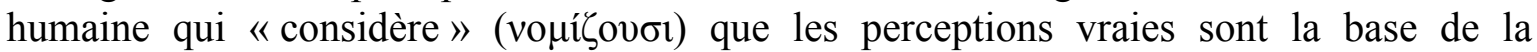
connaissance certaine. Bien entendu, Galien n'est pas un philosophe de profession et n'examine pas [120] les conséquences ultimes de sa conception : par exemple, la question de savoir comment seraient définies les perceptions vraies si la communauté de «tous les hommes » était composée de malades d'esprit ou de corps ne se pose pas ; on peut penser que Galien ne l'envisage pas pour la simple raison qu'il ne l'observe pas dans la réalité.

\footnotetext{
${ }^{45}$ Cf. 94, 14-16 Barigazzi (= I, 42, 11-14, 1 Kühn).

${ }^{46} \mathrm{Cf}$. Hypotyposes pyrrhoniennes, I, 113. Les traductions citées sont celles de Jean-Paul Dumont, Les Sceptiques grecs (textes choisis), PUF, Paris, 1966.

${ }^{47}$ Cf. 94, 16-22 Barigazzi (= I, 42, 14-43, 4 Kühn).
} 
Ce qui intéresse le médecin de Pergame, c'est de trouver un point de départ d'où il puisse construire un corpus de connaissances solides. Il observe la réalité et considère comme un axiome le consensus humain, de la même façon qu'un géomètre de l'Antiquité constate que deux droites parallèles ne se recoupent jamais, sans se poser la question de savoir s'il peut exister des espaces non euclidiens, simplement parce que l'espace euclidien est le seul qui soit observable dans la réalité quotidienne. Galien est un « homme de l'art», un $\tau \varepsilon \chi v i ́ \tau \eta \varsigma$, et à ce titre, il souhaite disposer d'outils intellectuels à valeur opératoire afin d'aller de l'avant, tandis que le Sceptique, de son côté, cherche toutes les occasions de faire obstruction à la marche de la pensée.

Or, explique Galien, il est nécessaire qu'il y ait des perceptions fausses et des vraies, de même qu'il est nécessaire que certaines catégories de personnes soient plus aptes à effectuer des perceptions vraies que d'autres. Cette discrimination est indispensable si l'on veut juger, car le jugement implique l'examen ou l'utilisation de perceptions qui le précèdent.

Voilà donc notre probabiliste pris au piège : s'il argumente dans un sens ou dans l'autre (peu importe lequel) il reconnaît implicitement l'existence des deux catégories du vrai et du faux. S'il refuse d'affirmer quelle est la position vraie et qu'il laisse à chacun le soin d'en juger par soi-même, il reconnaît implicitement à chacun la capacité de distinguer le vrai du faux. Donc le vrai et le faux existent, de l'aveu implicite du probabiliste ${ }^{48}$.

Si la connaissance de la vérité est possible, encore faut-il savoir par quels moyens y parvenir, quels outils discriminateurs ou « critères » utiliser pour séparer le vrai du faux.

Sur ce point, la position des Pyrrhoniens et des disciples d'Ar- césilas est nette : la nature n'a donné à l'homme aucun critère permettant de discerner le vrai et le faux, donc tout jugement [121] est impossible, donc il faut suspendre le jugement ${ }^{49}$. Cette argumentation est cohérente mais insatisfaisante.

À ce stade de la discussion, Galien interrompt son raisonnement pour examiner ce qui se passe concrètement dans la vie courante. La rupture est si brutale que Marquardt, dans son édition hypercritique, a conclu à une interpolation ${ }^{50}$. Mais ne serait-ce pas plutôt l'indice d'une rédaction un peu rapide de l'ouvrage, ou encore la volonté de prendre les Sceptiques à leur propre jeu, puisque ces derniers prétendent ${ }^{51}$ que la vie est le seul critère et qu'il faut « vivre selon les prescriptions et opinions communes »?

Or, que nous enseigne la vie ? Il existe certaines activités humaines, par exemple la gymnastique et les «arts » ou métiers techniques ( $\tau \dot{\varepsilon} \chi v \alpha \imath)$, dans lesquelles on ne perd pas son temps à disserter dialectiquement sur le vrai et le faux. Se former dans les activités techniques consiste, grâce à un entraînement, à corriger les gestes maladroits pour arriver à enchaîner sans la moindre faute les gestes corrects : donc on parvient au vrai par l'exercice.

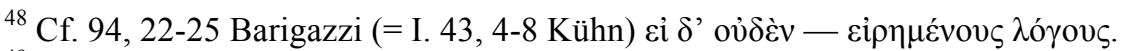

${ }^{49}$ Cf. 96, 5-8 Barigazzi (= I, 43, 17-44, 3 Kühn).

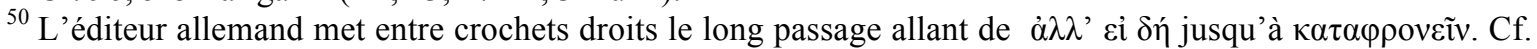
96, 8-20 Barigazzi (= I, 44, 3-45, 1 Kühn). On peut cependant observer qu'il y a dans le De optima doctrina plusieurs endroits où Galien rompt le fil de son discours pour opérer des digressions. Au début de l'opuscule, par exemple, en plein exposé sur les ouvrages de Favorinus, il ouvre une parenthèse pour reprocher au rhéteur d'Arles de ne pas s'exprimer dans un style purement attique lorsqu'il emploie les termes de " compréhension » et de " représentation compréhensive ».

${ }^{51}$ Cf. Sextus Empiricus, Hypotyposes pyrrhoniennes, II, 246.
} 
Pour atteindre ce but, l'étudiant n'est pas livré à lui-même, mais travaille sous la direction d'un maître qui s'abstient d'intervenir quand ses gestes sont corrects, et qui le corrige quand il commet une erreur : ce qui signifie que la possession du vrai est un acquis.

C'est alors que Galien enchaîne sur une deuxième remarque : tandis que les « hommes de l'art» prennent soin de ne pas détruire la confiance qu'ont leurs étudiants dans les «critères naturels" (les sens qui permettent de distinguer le bon du mauvais, le vrai du faux), mais leur apprennent à en faire bon usage, les Probabilistes, eux, s'efforcent de ruiner les propositions les plus évidentes et les plus unanimement admises. Ainsi, les raisonnements de Carnéade tentent de déstabiliser les propositions [122] mathématiques fondamentales, comme celle qui dit que deux grandeurs égales à une troisième sont égales entre elles. Le jugement de Galien envers ce comportement est sans appel : ceux qui dégagent des problèmes sans leur apporter de solution ne sont que des sophistes.

Ces deux digressions, qui ont rompu la marche linéaire de la démonstration galénique pour examiner la question en termes concrets, ont permis de dégager le terrain pour la suite de la discussion. La faculté dont nous disposons pour distinguer le vrai du faux est-elle innée ou résulte-t-elle d'un apprentissage? Si elle était innée, tout le monde serait d'accord sur tout. Or, comme tel n'est manifestement pas le cas, il faut un apprentissage (Galien parle d'une "méthode») qui nous permette de faire correctement usage de nos facultés naturelles (nos «critères naturels ») pour parvenir au vrai. Et la conclusion du raisonnement rejoint l'observation de la réalité : cette "méthode » est analogue à celle qu'emploient déjà les $\tau \varepsilon \chi v i ́ \tau \alpha$.

Tel est le second coup de boutoir qu'enfonce Galien, dans la muraille probabiliste : puisqu'il faut exercer ses facultés naturelles pour bien juger, on doit se demander comment les aider à remplir cette tâche, et non pas les paralyser ou les détruire en jetant le doute sur leur validité, ou encore, ce qui n'est guère mieux, laisser les gens faire usage de leurs facultés naturelles sans aucune préparation. Le Probabiliste est comme un artisan qui demanderait à son apprenti d'effectuer des mesures sans lui fournir ni équerre, ni balance, ni règle, ni compas ${ }^{52}$.

Quels sont les instruments naturels qui nous permettent de distinguer le vrai du faux ? Encore une fois, c'est l'observation de la réalité qui fournit la réponse, autorisant Galien à réfuter les Sceptiques en respectant la démarche recommandée par ces mêmes Sceptiques. Nous voyons les objets avec les yeux et nous percevons la saveur d'un fruit avec la langue : donc les différents sens servent, chacun dans leur domaine, à appréhender une réalité sensible. Et comme nous n'avons pas besoin d'un raisonnement supplémentaire pour nous assurer de cette réalité, nous avons affaire à une évidence sensible ${ }^{53}$. De la même façon, l'esprit est concerné par les évidences intelligibles. [123]

Afin d'éviter les sophismes ${ }^{54}$ qui proviennent du jugement des représentations sensibles par les concepts, Galien affirme que les réalités sensibles se jugent d'après la sensation, et les réalités intelligibles à l'aide de l'esprit ${ }^{55}$.

\footnotetext{
${ }^{52}$ Cf. 100, 6-8 Barigazzi (= I, 47, 1-4 Kühn).

${ }^{53}$ Cf. 102. 18-20 Barigazzi (= I, 49, 7-9 Kühn).

${ }^{54}$ Bien que Galien ne donne dans son De optima doctrina aucun exemple de conclusion erronée lorsqu'on juge dialectiquement d'une évidence sensible, on peut songer à un cas célèbre : Anaxagore disait que la neige est de l'eau gelée, or, l'eau est noire, donc la neige est noire. Cf. Sextus Empiricus, Hypotyposes pyrrhoniennes, I, 33.

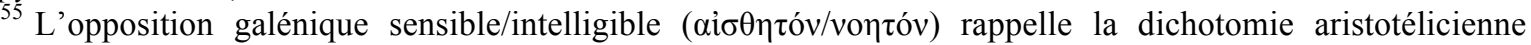

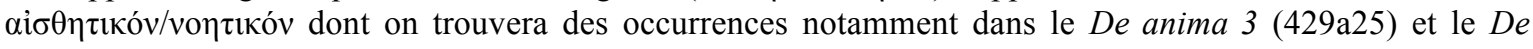


De plus, puisque nous nous servons de nos sens comme critères du vrai sensible dans

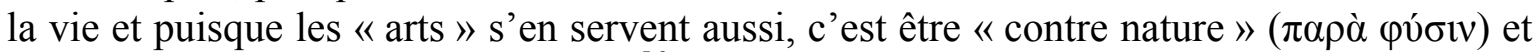
briser toute possibilité de discussion ${ }^{56}$ que de ne pas se fier à eux. Bref, l'existence du critère naturel est comme celle de l'axiome en géométrie : elle s'observe mais ne se démontre pas.

Le traité De praenotione ad Epigenem rapporte un incident survenu pendant une démonstration publique d'anatomi ${ }^{57}$. Au moment où Galien allait sectionner certains nerfs chez un animal pour démontrer que ceux-ci sont nécessaires à la production de la voix, le philosophe péripatéticien Alexandre, présent dans l'assistance, lui demanda si l'on pouvait accorder sa confiance aux données de la sensation. Ulcéré, le médecin de Pergame lâcha ses instruments et quitta la salle. Il voulait montrer par ce geste spectaculaire qu'il n'est pas possible de faire avancer la connaissance si l'on refuse de s'appuyer sur le témoignage des sens.

Mais que faire des choses qui ne relèvent ni de l'évidence sensible ni de l'évidence intelligible ? C'est là qu'intervient la notion de connaissance médiate. Une connaissance qui ne résulte pas directement de l'évidence s'obtient par le biais d'une autre. On a donc besoin d'une méthode permettant de distinguer l'évident du non évident et enseignant quelles opérations [124] de l'esprit mettre en œuvre dans ce dernier cas ${ }^{58}$. L'influence du modèle géométrique est manifeste ; on part d'évidences saisissables par tous (les axiomes), et l'on construit progressivement un système de propositions de moins en moins immédiatement saisissables et qui nécessitent des raisonnements et démonstrations de plus en plus complexes (les théorèmes). Ce n'est pas un hasard si Galien se représente le maître comme un personnage dont les yeux de l'esprit possèdent une acuité supérieure et qui guide dans la contemplation des choses ceux dont la vue est moins perçante. Il tire cette image de Platon ${ }^{59}$ qui était, lui aussi, fasciné par la méthode géométrique.

Par conséquent, on ne peut enseigner que si l'on possède au préalable une théorie solide de la connaissance. Il est tout à fait logique que Galien ait intitulé $D u$ meilleur enseignement un opuscule où il passe la majeure partie de son temps à réfuter l'antidogmatisme gnoséologique de Favorinus.

Les probabilistes sont souvent des professeurs, comme Favori- nus, voire des chefs d'école à l'exemple de Carnéade. Cette fonction implique le devoir de transmettre aux

generatione animalium 2 (736bl4). L'emploi de cette terminologie nous révèle une fois de plus que Galien garde ses distances aussi bien à l'égard des Sceptiques que de leurs adversaires stoïciens.

${ }_{56}$ Cf. 104, 1-5 Barigazzi (= I, 49, 10-11 Kühn).

${ }^{57}$ Cf. De praenotione ad Epigenem, XIV, 626, 17-630, 14 Kühn. Le passage est traduit par P. Moraux, op. cit., p. 81-84.

${ }^{58}$ Galien, qui a le sens de sa propre publicité, en profite pour signaler qu'il a rédigé un ouvrage traitant de ces

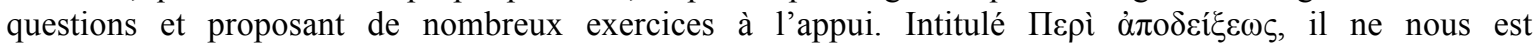
malheureusement pas parvenu.

${ }^{59}$ Il est impossible de retrouver, sous sa forme littérale, la citation faite par Galien dans les œuvres de Platon telles qu'elles nous sont parvenues. Appartient-elle à un dialogue aujourd'hui perdu, à un commentaire, ou encore Galien cite-t-il de mémoire en fusionnant des éléments épars? Au livre VII de la République, Socrate réfute les sophistes qui veulent introduire l'éducation dans l'âme «comme on donnerait la vue à des yeux aveugles » (518c), car le fait d'apprendre est en réalité une réminiscence. L'éducation est donc l'art de détourner les yeux de l'âme loin des ombres de la caverne pour les diriger vers le soleil que représente l'Idée $\mathrm{du}$ Bien. Cette éducation s'effectue avec l'aide de la méthode dialectique (533d) sous la direction des philosophes. Dans les Lois (715e), l'Athénien fait la remarque suivante, que nous citons dans la traduction d'É. des Places, C.U.F., Paris, 1965, tome XI, 2 : «C'est qu'en pareille matière [l'examen dialectique], tout homme dans sa jeunesse y voit le plus trouble $(\dot{\alpha} \mu \beta \lambda \hat{\tau} \tau \alpha \tau \alpha)$, mais sur le tard il y voit le plus clair (ó $\xi \dot{v} \tau \alpha \tau \alpha)$. 
étudiants des connaissances sûres et de leur apprendre les méthodes pour y parvenir. Or, bien loin de réaliser ce programme, Favorinus met en doute ce qu'il y a de plus certain (même l'existence du soleil n'est pas assurée) et entraîne ses étudiants à la pratique d'une méthode destructrice : la discussion généralisée du pour et du [125] contre qui, ne cherchant pas à établir une synthèse ni même à départager les opinions en présence, aboutit au sentiment qu'en fin de compte tout se vaut.

En outre, après avoir introduit chez ses étudiants l'habitude de douter des connaissances et des méthodes, il leur demande néanmoins de juger par eux-mêmes, alors que le rôle du jugement est précisément de lever le doute en s'appuyant sur des connaissances certaines et sur des raisonnements permettant d'atteindre la certitude. Comme l'écrit Galien ${ }^{60}$, «Favorinus ne semble agir à peu près comme un homme qui prétend que Dion est aveugle de nature, mais qu'il peut juger lequel d'entre nous a le teint plus sombre ou plus clair, sans songer que, lorsqu'on s'apprête à porter un tel jugement, on doit d'abord disposer de la vue. »

Galien dénonce deux tactiques frauduleuses qu'utilisent les Probabilistes. Ceux-ci appliquent aux données de la sensation (par exemple le fait de l'existence du soleil) les méthodes du raisonnement, et constatant que les résultants sont absurdes, cherchent à en conclure que le raisonnement est universellement invalide ${ }^{61}$. Ils s'efforcent aussi de mettre en œuvre le raisonnement après l'avoir amputé de tout moyen de fonctionner correctement (en le privant des critères de distinction entre le vrai et le faux), puis ils le discréditent en lui faisant soutenir indifféremment les thèses opposées avec une égale impuissance à faire pencher la décision dans un sens ou dans l'autre. [126]

L'attitude des professeurs probabilistes va à l'encontre de ce qu'on attend des enseignants. Ils n'améliorent pas leurs étudiants qu'ils rendent semblables à des " ânes » parce qu'il n'existe, sur le plan des résultats, aucune différence entre la privation d'intelligence et le refus de s'en servir sous prétexte qu'on ne lui fait pas confiance ${ }^{62}$.

En trahissant la fonction première de l'enseignement, les Probalistes le rendent inutile. Quand Favorinus laisse ses étudiants juger comme ils veulent des questions dont ils ont débattu le pour et le contre, il abolit la différence qui existe entre le maître et l'élève. $\mathrm{Du}$ reste, les méfaits de ce comportement rejaillissent sur leurs auteurs, puisque les étudiants qui veulent savoir quelque chose de certain touchant à la doctrine des Péripatéticiens, des Stoïciens ou d'une autre école s'adressent à un maître appartenant à l'école concernée, et n'ont que faire des opinions que les Probabilistes peuvent formuler à leur sujet ${ }^{63}$. L'exercice du doute sceptique ${ }^{64}$ finit par «envelopper dans ce doute

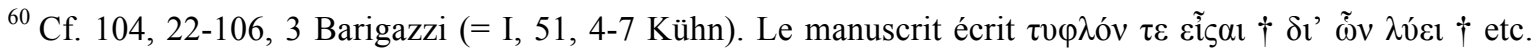

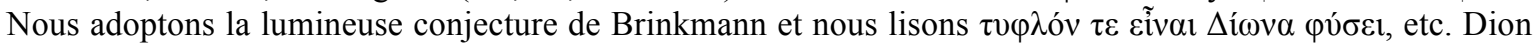
est le nom d'un personnage fictif qu'emploient les philosophes quand ils imaginent, pour illustrer leur démonstration, un exemple qui met en scène un individu type. Le nom apparaît souvent de concert avec celui de Théon, et plus rarement en compagnie de celui de Socrate, de Ménippe ou de Théétète. Les auteurs qui l'emploient le plus fréquemment dans son acception philosophique sont Alexandre d'Aphrodise (75 fois), Sextus Empiricus (39), Jean Philopon (24), Diogène 1.aërce (15 fois dans la section de la Vie de Zénon consacrée à la logique stoïcienne), Ammonius (9) et Épictète (7). Galien en fait lui-même usage soixantedouze fois, et la définition $\mathrm{n}^{\circ} \mathrm{CXV}$ de l'apocryphe Definitiones medicae cite Théon et Dion comme exemples d'êtres vivants doués de sensation.

${ }^{61} \mathrm{Cf}$. R. Van der Elst, De cujuslibet animi peccatorum dignotione atque medela. De propriorum animi cujuslibet affectuum dignotione et curatione, Dela- grave, Paris, 1914, n. 65, p. 107. Il faut cependant signaler que l'idée développée par Van der Elst ne se trouve nullement dans les Entretiens (I, 5) d'Épictète.

${ }^{62}$ Cf. 106, 3-9 Barigazzi (= I, 51, 8-15 Kühn).

${ }^{63}$ Cf. 94, 25-96, 4 Barigazzi (= I, 43, 8-16 Kühn).

${ }^{64}$ La remarque est de Van der Elst, op. cit., n. 65, p. 107-108.
} 
l'existence même du maître et de l'élève "), et remet en question, au passage, l'utilité de l'existence du professeur de scepticisme.

L'enjeu réel du bref opuscule que constitue le De optima doctrina dépasse la simple polémique individuelle avec Favorinus. Les points-clés développés par Galien sont l'existence d'évidences sensibles et intelligibles et la possibilité de construire un système de connaissances non immédiatement saisissables grâce à des raisonnements opérés sur la base de ces deux sortes d'évidences.

Le médecin de Pergame a toujours soutenu que la médecine repose sur l'anatomie, c'est-à-dire l'observation des constituants réels des êtres vivants. Les faits dégagés par ces observations préalables sont ensuite soumis à des raisonnements servant à expliquer le comment et le pourquoi des phénomènes médicaux.

Ce dessein grandiose, visant à arracher la médecine à son statut de technique pour la promouvoir au rang de science, devait se heurter à de nombreuses difficultés. Le penseur dogmatique qu'est Galien devait souffrir de ne pouvoir démontrer toutes les vérités du domaine médical comme on le fait en géométrie, et il [127] devait être ulcéré de voir qu'en maintes occasions, une simple observation valait mieux qu'un raisonnement.

C'est pourquoi il a jugé nécessaire de batailler contre toutes les formes du scepticisme philosophique, lequel lui semblait alimenter les conceptions anti-rationalistes et anti-étiologiques des Empiriques et des Méthodiques, écoles dont il a tant de fois dénoncé l'insuffisance voire la fausseté complète de la doctrine. 